\title{
Brightness-Temperature Retrieval Methods in Synthetic Aperture Radiometers
}

\author{
Ignasi Corbella, Senior Member, IEEE, Francesc Torres, Senior Member, IEEE, \\ Adriano Camps, Senior Member, IEEE, Nuria Duffo, Member, IEEE, and Mercè Vall-llossera, Member, IEEE
}

\begin{abstract}
Brightness-temperature retrieval techniques for synthetic aperture radiometers are reviewed. Three different approaches to combine measured visibility and antenna temperatures, along with instrument characterization data, into a general equation to invert are presented. Discretization and windowing techniques are briefly discussed, and formulas for reciprocal grids using rectangular and hexagonal samplings are given. Two known techniques are used to invert the equation, namely, inverse Fourier transform and $G$-matrix pseudoinverse. The proposed preprocessing approaches combined with these two inversion methods are implemented with real data measured by an airborne Y-shaped interferometric radiometer over land and water, and are compared. The images indicate that best results are obtained when inverting an incremental visibility obtained after substracting a term that includes the individual antenna temperatures, the physical temperatures of the receivers, and a flat-target response directly measured from cold-sky looks.
\end{abstract}

Index Terms-Brightness temperature image reconstruction, interferometry, synthetic aperture microwave radiometers.

\section{INTRODUCTION}

$\mathbf{T}$ HE USE of aperture synthesis for microwave Earthobservation radiometers was first proposed in 1983 by Le Vine and Good [1] as a way to overcome antenna size problems in high spatial resolution applications, particularly at low frequencies and spaceborne instruments. The first instrument using this concept was ESTAR, developed at NASA Goddard in the 1990s [2]. The first proposal of a two-dimensional (2-D) instrument was made by the European Space Agency (ESA) for the Soil Moisture and Ocean Salinity (SMOS) mission [3], and it led to the development of Microwave Imaging Radiometer with Aperture Synthesis (MIRAS) [4]. Airborne instruments using 2-D aperture synthesis were developed by the Helsinky University of Technology (HUT-2-D) [5] and by ESA/EADS-CASA [airborne MIRAS (AMIRAS)] [6] in 2006. A prototype for a 50-GHz 2-D instrument (GeoStar) was

Manuscript received January 18, 2008; revised April 8, 2008 and July 4, 2008. First published December 2, 2008; current version published December 17, 2008. This work was supported in part by the EADS - Casa Espacio and the European Space Agency (ESA) in the frame of the MDPP-3 and SMOS PLM phase C/D projects and in part by the Spanish MCYT and FEDER funds under Project TIC 2002-04451-C02-01 and Project TEC2005-06863-C02-01/TCM.

The authors are with the Remote Sensing Laboratory, Institut d'Estudis Espacials de Catalunya Centre de Recerca de l'Aeronàutica i de l'Espai/ Universitat Politècnica de Catalunya, Department of Signal Theory and Communications, Universitat Politècnica de Catalunya, 08034 Barcelona, Spain (e-mail: corbella@tsc.upc.edu; xtorres@tsc.upc.edu; camps@tsc.upc.edu; duffo@tsc.upc.es; merce@tsc.upc.edu).

Color versions of one or more of the figures in this paper are available online at http://ieeexplore.ieee.org.

Digital Object Identifier 10.1109/TGRS.2008.2002911 developed by the Jet Propulsion Laboratory [7] with application as a geosounder from a geostationary orbit. Increasing interests in interferometric aperture synthesis is being observed in the last years, and teams in Europe, USA, and China [8] are pushing this technology very fast. In this paper, general techniques for retrieving brightness-temperature maps from the inversion of measured visibility and antenna temperature in synthetic aperture radiometers are reviewed.

Effects specific to the SMOS case, such as extension of the alias-free region or mitigation of scene-dependent bias, are not covered here. They are treated in [9] and [10], where techniques to improve image reconstruction using a priori information are given. Good results were reported in these references by using end-to-end simulations to compare theoretical and retrieved brightness-temperature maps. These techniques are not in contradiction with the retrieval methods presented here and can be used whenever the needed a priori information is available.

On the other hand, this paper presents general approaches for brightness-temperature retrieval, which are applicable to any 2-D instrument, including SMOS. They are implemented on data measured by an airborne instrument (AMIRAS), and the resultant images are compared in order to assess the relative quality of the different approaches. The comparison is thus done on the basis of measured data, which is an important difference with respect to the results reported in [9].

\section{Antenna Temperature AND Visibility}

Antenna temperature and visibility are the primary measurements of interferometric aperture synthesis radiometers. The first one characterizes the thermal noise power collected by a single antenna, whereas the second one, the complex cross correlation between the signals collected by two antennas. Both have units of Kelvin and are related to the source brightness temperature by an integral in which the contributions of all the elementary radiators are combined weighted by the antenna patterns [11], [12]. In the case of the visibility, the time delay from a given source point to each of the two antennas provides a complex exponential term that is a function of the relative antenna spacing. Consequently, measuring the visibility with different pairs of antennas (baselines) is equivalent to measuring the Fourier transform of the brightness temperature as a function of antenna spacing. The brightness-temperature image is then retrieved by using a suitable inversion algorithm from multiple measurements of visibility at different spacings. However, differences in individual antenna patterns and decorrelation effects due to limited signal bandwidths make things more 
difficult. Moreover, noise coupled between adjacent antennas, which depends on the physical temperature of the receivers, is responsible for correlated output that is added to the one coming from the external target.

Interferometric aperture synthesis radiometers are formed by a significant number of baselines made of either physically different antennas or by moving a small number of elements. In any case, the visibility of a given baseline is measured by cross correlating, during a limited integration time, the complex analytic signals of the receivers' outputs. The contribution of the receivers themselves to the amplitude and phase of the correlations must be canceled by internal calibration. Inversion techniques always assume that these calibration processes have been applied to the raw data so that the input for inversion is shown in the following.

1) For all pairs of different antennas at positions $\left(x_{k}, y_{k}\right)$ and $\left(x_{j}, y_{j}\right)$ :

a) The visibility $V_{k j}\left(u_{k j}, v_{k j}\right)$, where $u_{k j}=\left(x_{j}-x_{k}\right) /$ $\lambda_{0}, v_{k j}=\left(y_{j}-y_{k}\right) / \lambda_{0}$ and $\lambda_{0}$, is the wavelength at the center frequency $f_{0}$.

b) The fringe-washing function shape [13] $\overline{\tilde{r}}_{k j}(\tau)$, where $\overline{\tilde{r}}_{k j}(0)=1$ by definition.

2) For at least one antenna, its antenna temperature $T_{A}$.

The following section provides a general equation relating these measured data to the scene brightness temperature.

\section{EQUATIONS FOR IMAGE RECONSTRUCTION}

The starting point to define algorithms for brightnesstemperature retrieval is to establish the equation to solve. The following two different situations must be distinguished: 1) the visibility of nonzero baselines; and 2) the visibility of zero-spacing baselines. Both are measured by different means, namely: the first one by correlation of receiver pairs and the second one by total power detection.

\section{A. Nonzero Baselines}

For any pair of different antennas, the function to use in the inversion procedure is the fully calibrated system visibility divided by the fringe-washing function at the origin $\tilde{r}_{k j}(0)$, which is, in turn, obtained in the calibration process [14]. According to Corbella et al. [11], for any two antennas labeled $k$ and $j$ with $k \neq j$, this is given by

$$
V_{k j}\left(u_{k j}, v_{k j}\right)=\iint_{\xi^{2}+\eta^{2}<1} T_{B_{k j}}^{\prime}(\xi, \eta) e^{-j 2 \pi\left(u_{k j} \xi+v_{k j} \eta\right)} d \xi d \eta
$$

where $\xi$ and $\eta$ are the director cosines referred to the instrument geometrical (spherical) coordinate frame $(\xi=x / r, \eta=y / r)$ and $T_{B_{k j}}^{\prime}$ is the modified brightness temperature

$$
T_{B_{k j}}^{\prime}=\left(T_{B}(\xi, \eta)-T_{r_{k j}}\right) \operatorname{AP}_{k j}(\xi, \eta) \overline{\tilde{r}}_{k j}\left(-\frac{u_{k j} \xi+v_{k j} \eta}{f_{0}}\right)
$$

where $T_{B}(\xi, \eta)$ is the brightness temperature of the scene, $T_{r_{k j}}$ is the average backward noise temperature of the receivers, ${ }^{1}$ and $\operatorname{AP}_{k j}(\xi, \eta)$ is a function of antenna patterns defined as

$$
\operatorname{AP}_{k j}(\xi, \eta)=\frac{F_{n k}(\xi, \eta) F_{n j}^{*}(\xi, \eta)}{\sqrt{1-\xi^{2}-\eta^{2}} \sqrt{\Omega_{k} \Omega_{j}}}
$$

where $F_{n k, j}$ represents the normalized field antenna patterns and $\Omega_{k, j}$ represents the antenna equivalent solid angles. It is implicit in this formulation that all antennas have exactly the same polarization or, equivalently, that all polarization unit vectors have the same orientation at all directions. Then, the brightness temperature in (2) is the one corresponding to this particular polarization basis, which, in general, is rotated in a direction-dependent angle with respect to the standard horizontal or vertical polarizations on ground. On the other hand, if the two antennas forming the baseline have orthogonal polarizations, the term $-T_{r_{k j}}$ in (2) should not be included, and $T_{B}(\xi, \eta)$ becomes the complex polarimetric brightness temperature defined in the frame given by the two antenna polarization vectors. A more complete formulation for antennas having both copolar and cross-polar patterns, with respect to a fixed reference frame, can be found in [15]; however, this paper uses the simpler formulation in order to put emphasis on the inversion methods, which are applicable in all situations.

The visibility (1) is measured for all pairs of antennas producing a total of $N(N-1) / 2$ different complex values, where $N$ is the total number of antennas. By convention, they correspond to the pairs having $k<j$. The complete coverage in the $(u, v)$ plane is obtained by applying the hermitic property

$$
V_{j k}\left(u_{j k}, v_{j k}\right)=V_{k j}^{*}\left(u_{k j}, u_{k j}\right)
$$

obtaining $N(N-1)$ visibilities, of which some are redundant, meaning that they have the same values of $u$ and $v$. The degree of redundancy depends on the layout of the antennas on the mechanical structure.

\section{B. Flat-Target Response}

A "flat target" is defined as a completely unpolarized target having an equal brightness temperature from any direction [16]. Examples are anechoic chambers or the cosmic background radiation. For such a target having a temperature $T_{\mathrm{FT}}$, the corresponding visibility is given by (1) after substituting the constant value $T_{\mathrm{FT}}$ for $T_{B}(\xi, \eta)$ in (2)

$$
V_{k j}^{\mathrm{FT}}\left(u_{j k}, v_{j k}\right)=\left(T_{\mathrm{FT}}-T_{r_{k j}}\right) \mathrm{FTR}_{k j}\left(u_{k j}, v_{k j}\right)
$$

where

$$
\begin{array}{r}
\operatorname{FTR}_{k j}=\iint_{\xi^{2}+\eta^{2}<1} \operatorname{AP}_{k j} \overline{\tilde{r}}_{k j}\left(-\frac{u_{k j} \xi+v_{k j} \eta}{f_{0}}\right) \\
\times e^{-j 2 \pi\left(u_{k j} \xi+v_{k j} \eta\right)} d \xi d \eta
\end{array}
$$

\footnotetext{
${ }^{1}$ This is equal to the physical temperature if input isolators are placed in the receivers.
} 


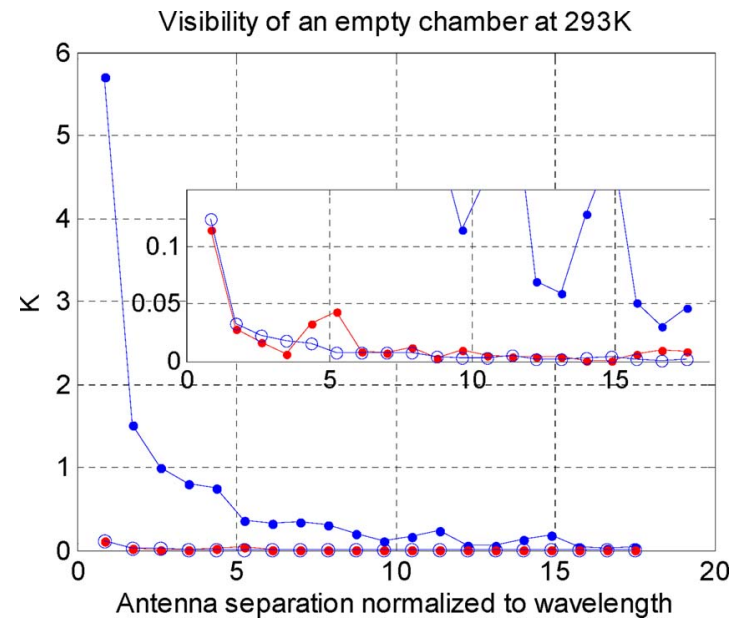

Fig. 1. Visibility of an anechoic chamber at $T_{\mathrm{FT}}=293 \mathrm{~K}$ corresponding to the 20 baselines formed by the antenna at the far edge of one of the arms and the rest of the antennas in the same arm of MIRAS. (Circles) Computed from (5). (Blue dots) Term due to only the chamber's temperature $\left(T_{\mathrm{FT}} \mathrm{FTR}_{k j}\right)$. (Red dots) Actual measurements.

is the flat-target response, which is a function only of the instrument. When a constant source is considered, the general formulation using copolar and cross-polar antenna patterns becomes simple, and it reduces the use of the scalar product of the two vector antenna patterns $\vec{F}_{n k} \cdot \vec{F}_{n j}$ in the numerator of (3). Note that, since the antenna solid angle is the integral of the power pattern in the whole space, $\mathrm{FTR}_{k k}=1$.

Fig. 1 shows the visibility of an anechoic chamber at $T_{\mathrm{FT}}=$ $293 \mathrm{~K}$ for the case of MIRAS. The circles are the computed values using (5), and the solid dots represent the term due only to the chamber temperature $T_{\mathrm{FT}} \mathrm{FTR}_{k j}$. In both cases, the flat-target response was analytically computed using (6) from antenna pattern measurements provided by Denmark University of Technology. Finally, the dots inside (or close to) the circles show the measurements obtained during the image validation tests that took place in the anechoic chamber of European Space Research and Technology Centre (ESTEC) with the fully deployed instrument inside it. The small discrepancy seen around the $5 \lambda$ separation coincides with the presence of a hinge separating two sections of the instrument. Based on the results shown in Fig. 1, it follows that the flat-target response can be neglected (i.e., $\mathrm{FTR}_{k j} \approx 0$ ) in the case of large antenna spacings.

The flat-target response can be computed from antenna pattern measurements, or alternatively, it can be measured using a known flat target. In any case, once it is known, it can be used to cancel the term of a receiver temperature in the visibility by inverting the function $V_{k j}+T_{r_{k j}} \mathrm{FTR}_{k j}$. This is equivalent to using the same equations (1) and (2) but without the term $-T_{r_{k j}}$. Note that this approach was proposed in [14, eq. (24)] $\left[-V_{R}\right.$ term] and [9, eq. (7)] $\left[-V_{R}^{p q}\right]$, where the computation of the flat-target response from antenna patterns was proposed.

\section{Incremental Brightness Temperature and Visibility}

The integrated brightness temperature of the scene weighted by the antenna power pattern is the antenna temperature $T_{A}$.
Image reconstruction algorithms may take advantage of this by inverting the incremental brightness temperature defined as $\Delta T_{B}(\xi, \eta)=T_{B}(\xi, \eta)-T_{A}$. For a given baseline $(k, j)$, the modified incremental brightness temperature is conveniently defined by an equation similar to (2) but with the antenna temperature replacing the receiver backward noise temperature

$$
\begin{aligned}
\Delta T_{B_{k j}}^{\prime}=\left(T_{B}(\xi, \eta)\right. & \left.-T_{A_{k j}}\right) \\
& \times \operatorname{AP}_{k j}(\xi, \eta) \overline{\tilde{r}}_{k j}\left(-\frac{u_{k j} \xi+v_{k j} \eta}{f_{0}}\right)
\end{aligned}
$$

where $T_{A_{k j}}=0.5\left(T_{A_{k}}+T_{A_{j}}\right)$ is the average of the antenna temperatures of both antennas, each one given by

$$
T_{A_{k}}=\iint_{\xi^{2}+\eta^{2}<1} T_{B}(\xi, \eta) \mathrm{AP}_{k k}(\xi, \eta) d \xi d \eta .
$$

The corresponding incremental visibility is defined directly by using (7) in (1)

$$
\Delta V_{k j}\left(u_{k j}, v_{k j}\right)=\iint_{\xi^{2}+\eta^{2}<1} \Delta T_{B_{k j}}^{\prime} e^{-j 2 \pi\left(u_{k j} \xi+v_{k j} \eta\right)} d \xi d \eta
$$

which, using the flat-target response, can be expressed as

$$
\Delta V_{k j}=V_{k j}-\left(T_{A_{k j}}-T_{r_{k j}}\right) \mathrm{FTR}_{k j} .
$$

The procedure, in this case, consists of first computing $\Delta V_{k j}$ from (10) and then inverting (7) and (9) to get $\Delta T_{B}$. Finally, the brightness temperature is obtained by adding the antenna temperature $T_{B}=\Delta T_{B}+T_{A}$. This procedure is equivalent to using the flat-target transformation [16] with a reference temperature equal to the measured antenna temperature. The main advantage of this approach is that the uncertainties of antenna pattern and fringe-washing function scale with the difference $T_{B}-T_{A}$, as is apparent from (7). Then, when imaging far away from coastlines, for example, in open ocean or large land areas, this difference is reduced, and the impact of the antenna measurement errors using this approach is minimum. This is confirmed by the results of Section VI.

\section{Zero Baselines}

The visibility measured by correlating the signals out of receiver pairs does not provide the value for the center of the $(u, v)$ plane. For mathematical consistency, this must be equal to the function used in the inversion particularized to the origin. Putting $u_{k j}=v_{k j}=0$ and $k=j$ in (1)-(3), it follows that

$$
V_{k}(0,0)=\iint_{\xi^{2}+\eta^{2}<1}\left(T_{B}(\xi, \eta)-T_{r_{k}}\right) \operatorname{AP}_{k k}(\xi, \eta) d \xi d \eta
$$

which, using (8), can be put simply as

$$
V_{k}(0,0)=T_{A_{k}}-T_{r_{k}} .
$$

If the $-T_{r}$ term is canceled before inversion, as it is proposed at the end of Section III-B, then the visibilities in the origin 
TABLE I

SUMMARY OF INVERSION APPROACHES

\begin{tabular}{|c|c|c|c|}
\hline$\#$ & $V\left(u_{k j}, v_{k j}\right)$ for $u_{k j}, v_{k j} \neq 0$ & $V(0,0)$ & $T(\xi, \eta)$ \\
\hline 1 & $V_{k j}$ & $T_{A_{k}}-T_{r_{k}}$ & $T_{B}(\xi, \eta)-\bar{T}_{r}$ \\
2 & $V_{k j}+T_{r_{k j}} \mathrm{FTR}_{k j}$ & $T_{A_{k}}$ & $T_{B}(\xi, \eta)$ \\
3 & $V_{k j}-\left(T_{A_{k j}}-T_{r_{k j}}\right) \mathrm{FTR}_{k j}$ & 0 & $T_{B}(\xi, \eta)-\bar{T}_{A}$ \\
\hline
\end{tabular}

become simply $T_{A_{k}}$. Finally, using (10) and taking into account that $\mathrm{FTR}_{k k}=1$, it follows that the incremental visibility is null at the origin $\Delta V_{k}(0,0)=0$. Note that this is basically the criterion used to define the "differential visibilities" in [14] and [9]. However, in these references, the terms added to achieve $\Delta V(0,0)=0$ are analytically computed from the visibility equation assuming known antenna patterns and fringe-washing functions, as well as a priori information on the scene. Here, the antenna temperatures of the front ends, as well as the flattarget response, are directly used, which are theoretically the same but conceptually simpler.

In any case then, antenna temperatures need to be known. These can be provided if all receivers in the array are calibrated as total power radiometers. Note that the antenna temperatures are slightly different from one receiver to another. Although this seems to be the best strategy, in SMOS, only three antenna temperatures are measured - those corresponding to three well-calibrated noise injection radiometers included near the center of the array for this purpose. The average of all three measurements is used as the unique antenna temperature.

\section{E. Approach Summary}

In summary, image reconstruction consists of solving for $T(\xi, \eta)$ in an equation of the type $V(u, v)=\mathcal{F}[T(\xi, \eta)]$, where $V(u, v)$ is known. This can be written as

$$
V\left(u_{k j}, v_{k j}\right)=\int_{-\infty}^{\infty} \int_{-\infty}^{\infty} T_{k j}^{\prime}(\xi, \eta) e^{-j 2 \pi\left(u_{k j} \xi+v_{k j} \eta\right)} d \xi d \eta
$$

with

$$
T_{k j}^{\prime}= \begin{cases}T(\xi, \eta) \mathrm{AP}_{k j}(\xi, \eta) \overline{\tilde{r}}_{k j}\left(-\frac{u_{k j} \xi+v_{k j} \eta}{f_{0}}\right), & \xi^{2}+\eta^{2}<1 \\ 0, & \xi^{2}+\eta^{2} \geq 1\end{cases}
$$

in which, for consistency, $T(\xi, \eta)$ should only be a function of the director cosines $\xi$ and $\eta$ but not of the specific baseline used. Based on the results of the previous sections, there are three approaches in the choice of $V$ and $T$, which are summarized in Table I and described in the following.

1) Approach \#1 uses the visibility directly as obtained from the calibration procedure. To take into account the $-T_{r}$ term, this must be subtracted both at the zero visibility and at the brightness temperature. Note that the term subtracted to $T_{B}$ consists of the average receiver temperature $\bar{T}_{r}$ in order to comply with the requirement that $T$ does not depend on the specific baseline.
2) In approach \#2, the $-T_{r}$ term is canceled in the visibility before inversion. In this case, the zero visibility is directly the antenna temperature, and the retrieved variable is simply the brightness temperature.

3) Finally, approach \#3 inverts the incremental visibility (10). Then, the zero visibility vanishes, and the retrieved function is the difference between the brightness and the average antenna $\bar{T}_{A}$ temperatures.

In any case, the flat-target response is assumed to be known, either from direct measurement using, for example, the cold sky or computed from measured antenna patterns using (6). Alternatively, it can be neglected using simply $\mathrm{FTR}_{k j}=0$, which may be a good option for instruments having large antenna separations in terms of the wavelength (Fig. 1).

\section{DisCRETIZATION AND WINDOWING}

The visibility in (13) is measured only at the discrete points $\left(u_{k j}, v_{k j}\right)$. Their specific location in the $(u, v)$ plane depends on the particular layout of the antennas in the array. For example, in a Y-shaped instrument, they are placed in a hexagonal grid and form a star shape [17] (Fig. 2). In a U-shaped instrument [5], the grid is rectangular, and other geometries, for example, circular [8], have different distributions of points. After inversion, the brightness temperature is obtained also in a limited number of points in the director cosines domain $(\xi, \eta)$. In principle, the choice of the $(\xi, \eta)$ grid should be irrelevant. However, efficient inversion methods require the use of a grid reciprocal to the one in $(u, v)$ [17]. This is done by making a change of variables from $(u, v)$ to $\left(k_{1}, k_{2}\right)$ and from $(\xi, \eta)$ to $\left(n_{2}, n_{1}\right)$, in which both $\left(k_{1}, k_{2}\right)$ and $\left(n_{2}, n_{1}\right)$ are identical regular grids of $N_{T} \times N_{T}$ integers, where $N_{T}^{2}$ is the number of nonredundant $(u, v)$ points. In the case of a Yshaped instrument, this is also the total number of points in the smallest hexagon containing the star of measured points (Fig. 2). Table II shows the change of variables applicable to both rectangular (U-shaped) and hexagonal (Y-shaped) grids for instruments having unit antenna spacing referred to wavelength equal to $d$. In the Y-shaped case, the formulas assume that one of the arms is in the $(-x)$ direction. In all cases, the value of $N_{T}$ is related to the number of antennas in each arm $N_{\mathrm{EL}}$ by the formulas given.

The limited extent of the visibility domain in the $(u, v)$ plane is interpreted as a multiplication by a "rectangular" window, which is equal to unity for the measured points and is zero for the rest. As a standard practice in digital signal processing to improve sensitivity at the expense of spatial resolution and to reduce ripples in high-contrast images, other windowing functions can be used. For example, applying the Blackman window consists of multiplying the visibility by the function

$$
W(u, v)=0.42+0.5 \cos \left(\frac{\pi \rho}{\rho_{\max }}\right)+0.08 \cos \left(\frac{2 \pi \rho}{\rho_{\max }}\right)
$$

where $\rho=\sqrt{u^{2}+v^{2}}$ and $\rho_{\max }$ its maximum value. The window can be applied directly to the visibility or to the 

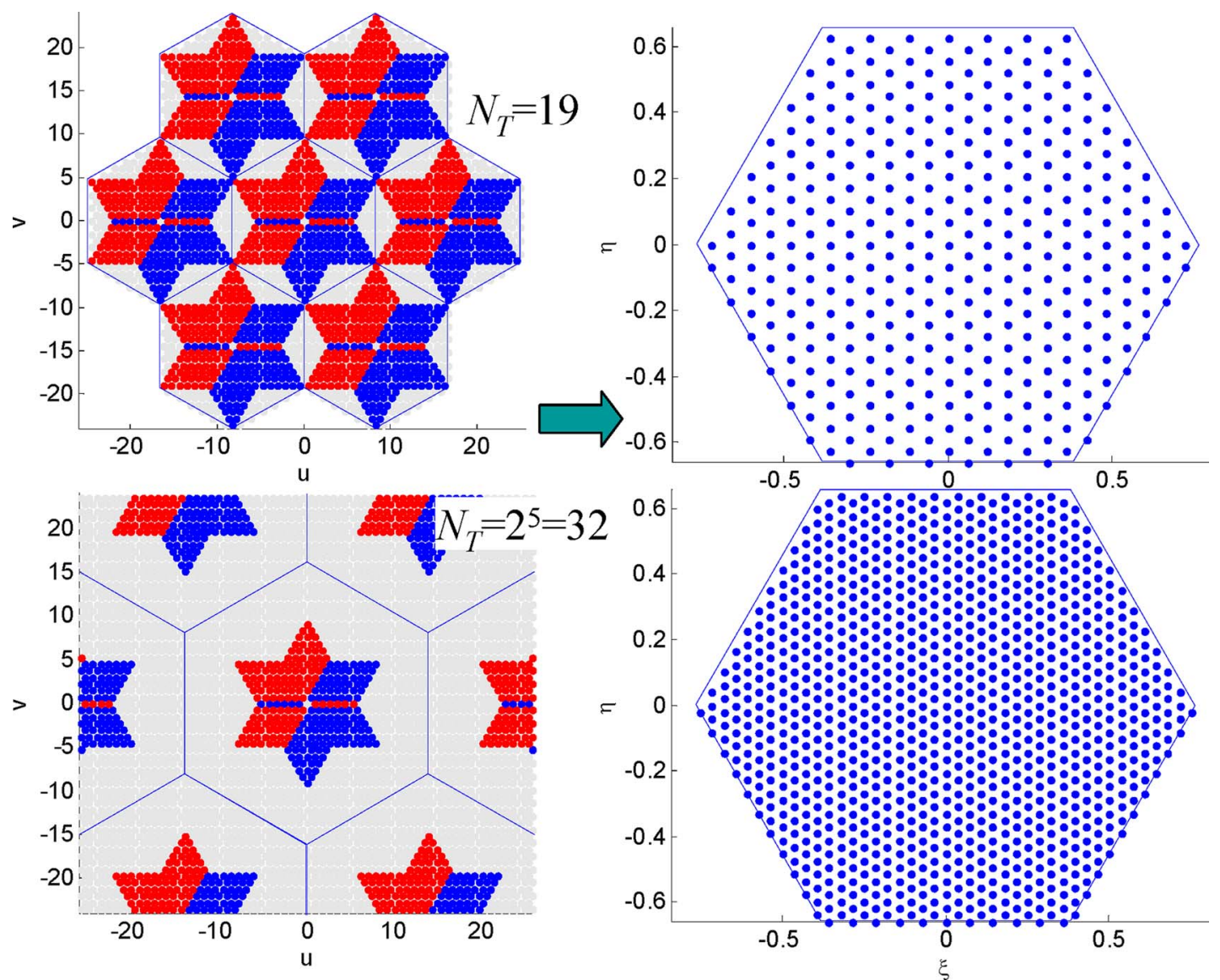

Fig. 2. Effect of zero padding in the $(u-v)$ and $(\xi, \eta)$ coverage for a Y-shaped instrument of six elements per arm.

TABLE II

RECIPROCAL GRIDS DEFINITIONS

\begin{tabular}{|l|l|}
\hline \multicolumn{1}{|c|}{ Rectangular grid } & \multicolumn{1}{c}{ Hexagonal grid [17] } \\
\hline$u=k_{1} d$ & $u=\left(k_{1}-k_{2}\right) d / 2$ \\
$v=k_{2} d$ & $v=k_{2} d \sqrt{3} / 2$ \\
\hline$\xi=n_{2} /\left(N_{T} d\right)$ & $\xi=n_{2} /\left(N_{T} d\right)$ \\
$\eta=n_{1} /\left(N_{T} d\right)$ & $\eta=\left(2 n_{1}+n_{2}\right) /\left(\sqrt{3} N_{T} d\right)$ \\
\hline$N_{T}=2 N_{E L}+1$ & $N_{T}=3 N_{E L}+1$ \\
\hline
\end{tabular}

brightness temperature obtained after inversion with the rectangular window. This last option is the one used in the SMOS official data processing to allow users of brightness-temperature data to apply different windows.

After making the change of variables of Table II and considering only the discrete points available, (13) is rewritten as

$$
V\left(k_{1}, k_{2}\right)=\frac{1}{N_{T}^{2} \Delta S} \sum_{N_{m}}^{N_{M}} \sum_{N_{m}}^{N_{M}} T_{k j}^{\prime}\left(n_{2}, n_{1}\right) e^{-j \frac{2 \pi}{N_{T}}}\left(k_{1} n_{2}+k_{2} n_{1}\right)
$$

where $\Delta S$ is the elementary area in $u-v$, equal to $d^{2}$ for rectangular grids and $d^{2} \sin 60^{\circ}$ for hexagonal grids, and where the limits of the summations are

$$
\left[N_{m}, N_{M}\right]= \begin{cases}{\left[-\frac{N_{T}}{2}, \frac{N_{T}}{2}-1\right],} & N_{T} \text { even } \\ {\left[-\frac{N_{T}-1}{2}, \frac{N_{T}-1}{2}\right],} & N_{T} \text { odd }\end{cases}
$$

\section{INVERSION TECHNIQUES}

\section{A. Inverse Fourier Transform}

Equation (16) is a discrete Fourier transform only in the case that $T^{\prime}$ is solely a function of $n_{2}$ and $n_{1}$. From (14), this is achieved if the decorrelation due to the fringe-washing function is neglected $\left(\overline{\tilde{r}}_{k j} \approx 1\right)$ and if a unique average antenna pattern is used. An assessment of the impact of antenna errors in the retrieval for the MIRAS case can be found in [18] and [19]. After averaging all redundant visibilities, the Fourier inversion of (16) is

$$
T^{\prime}\left(n_{2}, n_{1}\right)=\Delta S \sum_{N_{m}}^{N_{M}} \sum_{N_{m}}^{N_{M}} V\left(k_{1}, k_{2}\right) e^{j \frac{2 \pi}{N_{T}}\left(k_{1} n_{2}+k_{2} n_{1}\right)}
$$

and the brightness temperature is readily obtained using (14) and the formulas given in Table I. If, for example, approach \#3 is used

$$
T_{B}(\xi, \eta)=\frac{T^{\prime}(\xi, \eta)}{\operatorname{AP}(\xi, \eta)}+\bar{T}_{A}
$$

where $\xi$ and $\eta$ are computed from $n_{2}$ and $n_{1}$ with the formulas of Table II and $\operatorname{AP}(\xi, \eta)$ is an average value of (3) across all antennas. It can be computed by introducing, in (3), the average of the antenna power patterns normalized to solid angle. Alternatively, it can be estimated from the flat-target response by the direct inversion of (6). Considering that the 


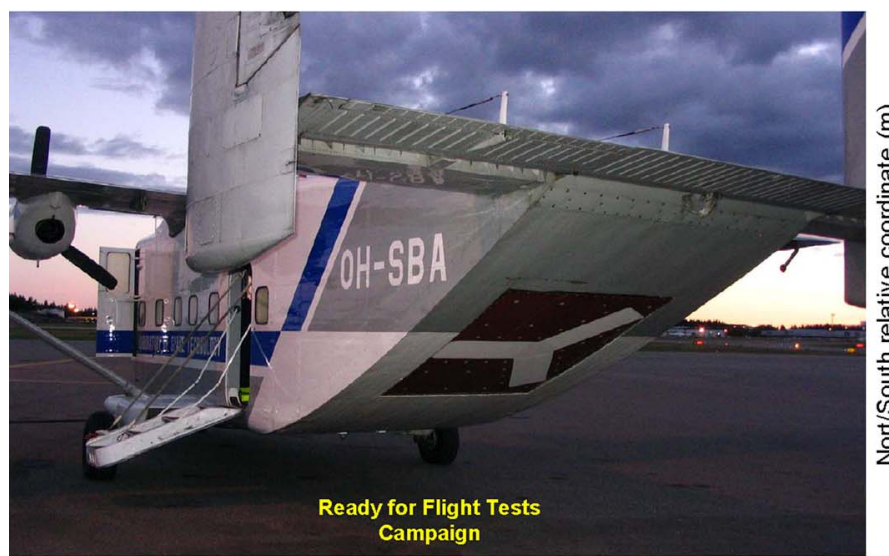

Fig. 3. (Left) AMIRAS installed on HUT SkyVan. (Right) Series of snapshots of vertical brightness temperature at antenna frame over the Pensaari island in Lohja Lake (Finland).

fringe-washing function is neglected in this method, this is equivalent to using (18) once again

$$
\operatorname{AP}\left(n_{2}, n_{1}\right)=\Delta S \sum_{N_{m}}^{N_{M}} \sum_{N_{m}}^{N_{M}} \operatorname{FTR}\left(k_{1}, k_{2}\right) e^{j \frac{2 \pi}{N_{T}}\left(k_{1} n_{2}+k_{2} n_{1}\right)} .
$$

This approach has the advantage that there is no need to perform any characterization of the individual antennas. A measurement of the flat-target response is the only instrumental characterization that is needed.

Fourier inversion is simple and efficient, particularly if a radix-2 fast Fourier transform (FFT) algorithm is used. In this case, $N_{T}$ must be a power of two, and this is achieved by extending the $(u, v)$ domain and putting $V=0$ in the new points. Considering that the visibility aliases become more separated from each other, the grid in $(\xi, \eta)$ becomes finer, resulting in an interpolation of the brightness temperature. Obviously, this does not mean that the spatial resolution is improved because this depends on the maximum distance between antennas. Fig. 2 shows an example of the visibility zero padding effect for a Y-shaped instrument having six elements per arm.

\section{B. Matrix Inversion}

Equation (16) is a linear system of equations, and it can be solved using any suitable mathematical method. In particular, it can be written in matrix form $[20]$ as $[V]=[G][T]$, where the elements of $[G]$ are given by

$$
G_{l m}=\frac{\operatorname{AP}_{k j}\left(n_{2}, n_{1}\right)}{N_{T}^{2} \Delta S} \overline{\tilde{r}}_{k j}\left(-\frac{k_{1} n_{2}+k_{2} n_{1}}{N_{T} f_{0}}\right) e^{-j \frac{2 \pi}{N_{T}}\left(k_{1} n_{2}+k_{2} n_{1}\right)}
$$

where the mapping between the subscripts $l, m$, and the indexes $k_{1}, k_{2}$ and $n_{2}, n_{1}$ depends on the equation arrangement used to construct the matrix, which is irrelevant. The matrix $[G]$ has as many rows as the visibility measurements, including the hermitic points (4) and the zero baseline. Redundant visibilities (including also those of zero spacing) and corresponding matrix elements should be averaged to avoid singularity problems when the matrix has several almost identical rows. After this averaging, the number of rows is equal to $6 N_{\mathrm{EL}}^{2}+6 N_{\mathrm{EL}}+1$ for a Y-shaped instrument and $\left(2 N_{\mathrm{EL}}+1\right)^{2}$ for a $\mathrm{U}$-shaped instrument, where $N_{\mathrm{EL}}$ is the number of elements in an arm. On the other hand, the number of columns is equal to the number of $(\xi, \eta)$ points in the selected grid. From (17), this is equal to $N_{T}^{2}$, and its minimum value, based on Table II, is $\left(3 N_{\mathrm{EL}}+1\right)^{2}$ for Y-shaped instruments and $\left(2 N_{\mathrm{EL}}+1\right)^{2}$ for $\mathrm{U}$-shaped instruments. Increasing the number of points produces the same interpolation effect as in the Fourier transform shown in Fig. 2.

For a Y-shaped instrument, the $G$ matrix is not square, and inversion has to be computed using a minimization algorithm in the least square sense, for example, the resolving matrix approach [21], selected as the official method for SMOS level-1 processing. However, the results given in the following section use the Moore-Penrose pseudoinverse directly provided by Matlab. Considering that AMIRAS is a small instrument, this approach is numerically efficient and has given good results.

\section{Amiras Brightness-Temperature Retrievals}

The methods presented in the previous sections have been implemented for the retrieval of brightness temperature using data measured by the small AMIRAS [6]. Fig. 3 shows a photograph of the instrument installed onboard the HUT SkyVan ready to start a flight. At right, a sequence of snapshots obtained during an overpass of an island over the lake Lohja in Finland is shown. They correspond to the vertical brightness temperature at the antenna frame imaged in the alias-free field of view. The snapshot marked with an arrow and those corresponding to the center of the lake are used as examples for analyzing the quality 

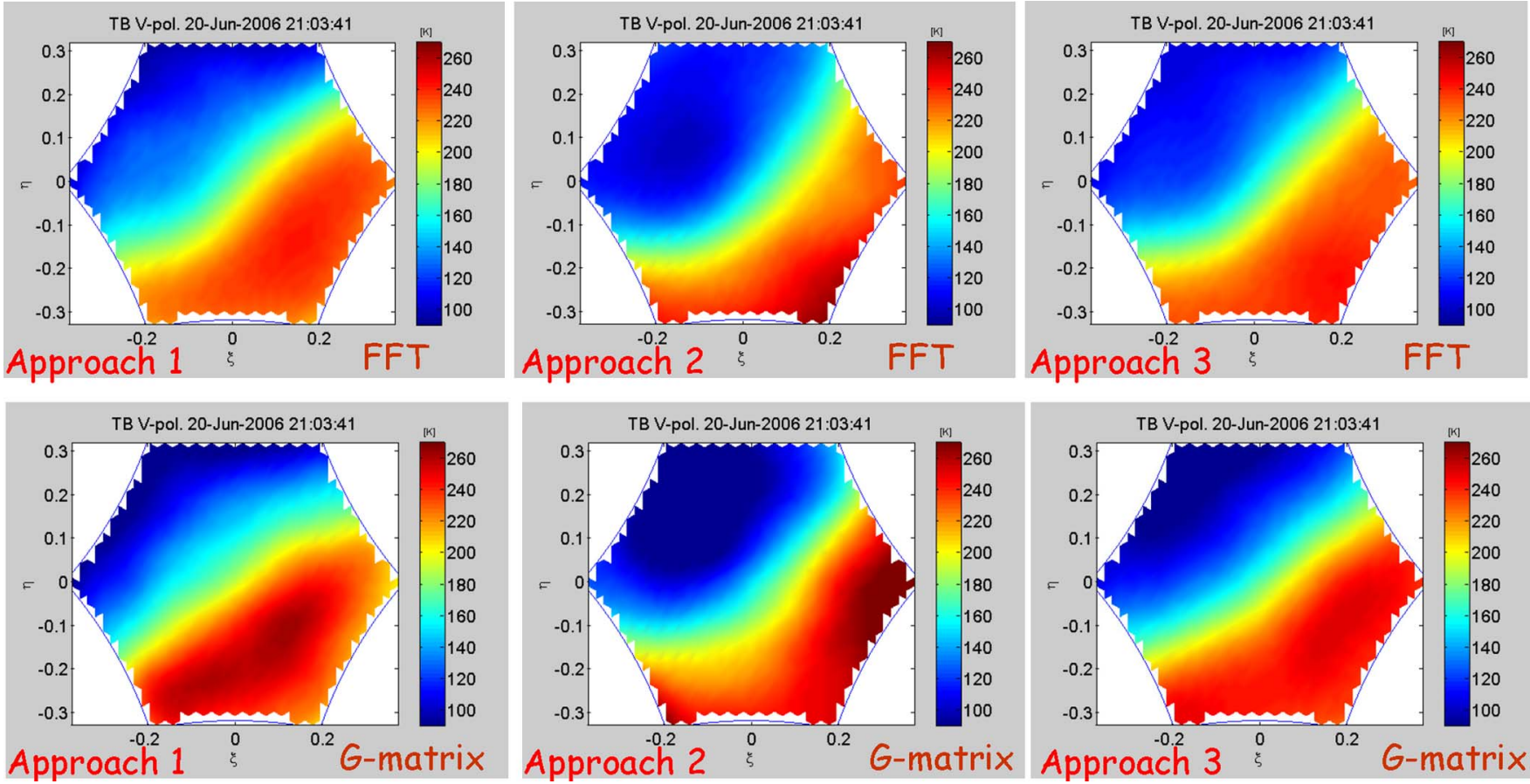

Fig. 4. Comparison of image reconstruction approaches using the snapshot marked with an arrow in Fig. 3. The horizontal flip is due to use of antenna ( $\xi, \eta)$ coordinates instead of distances on ground.
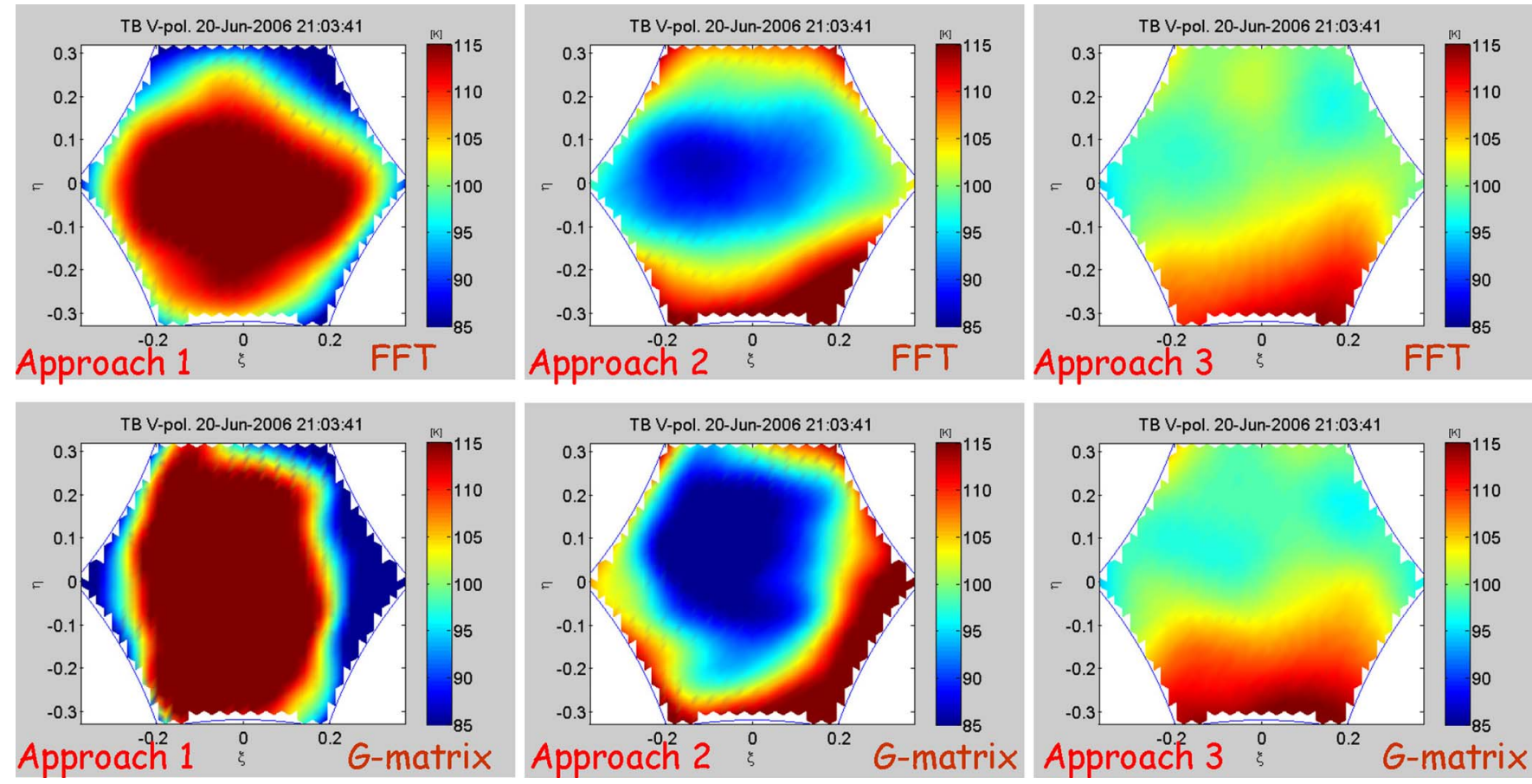

Fig. 5. Comparison of image reconstruction approaches using a snapshot of only fresh water in $V$ polarization. Flat-target response from Sky measurement is used in all cases.

of inversion using different approaches. Both qualitative and quantitative results are presented.

\section{A. Qualitative Results}

Fig. 4 shows a comparison between different imaging strategies. The three approaches given in Table I are used both in Fourier and matrix inversion using a flat-target response measured from sky view. The visual conclusion is that all methods provide consistent images and that little difference is seen when comparing the Fourier and $G$ matrix. To have more insight in the differences, a snapshot corresponding to the middle of the lake (only fresh water) has also been analyzed. The result is shown in Fig. 5. Here, it is clear that the only 


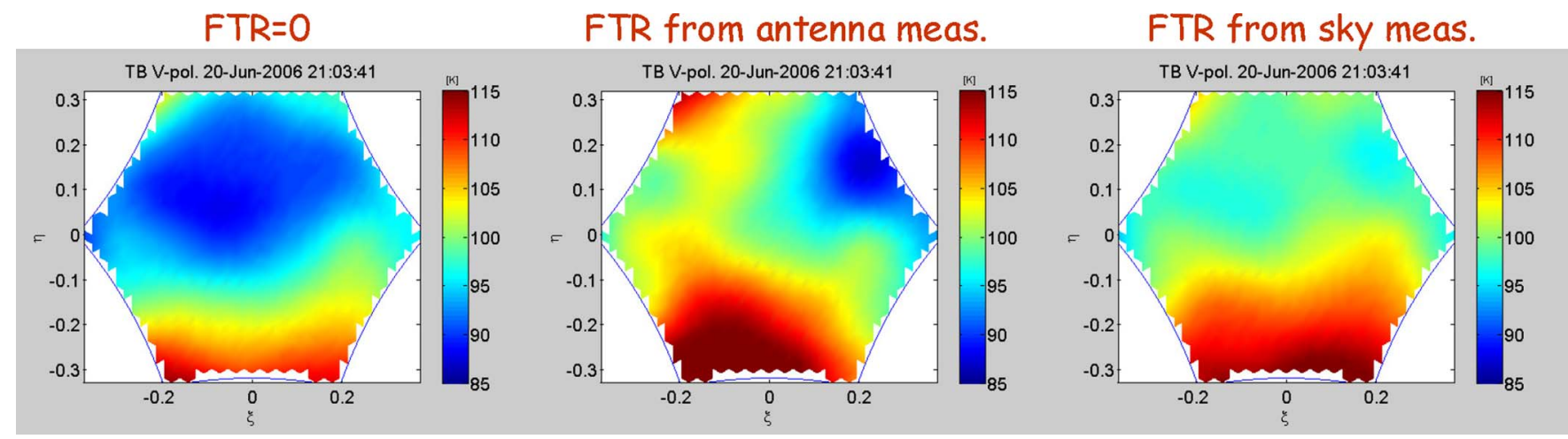

Fig. 6. Flat-target response choice in $G$-matrix inversion and approach \#3.

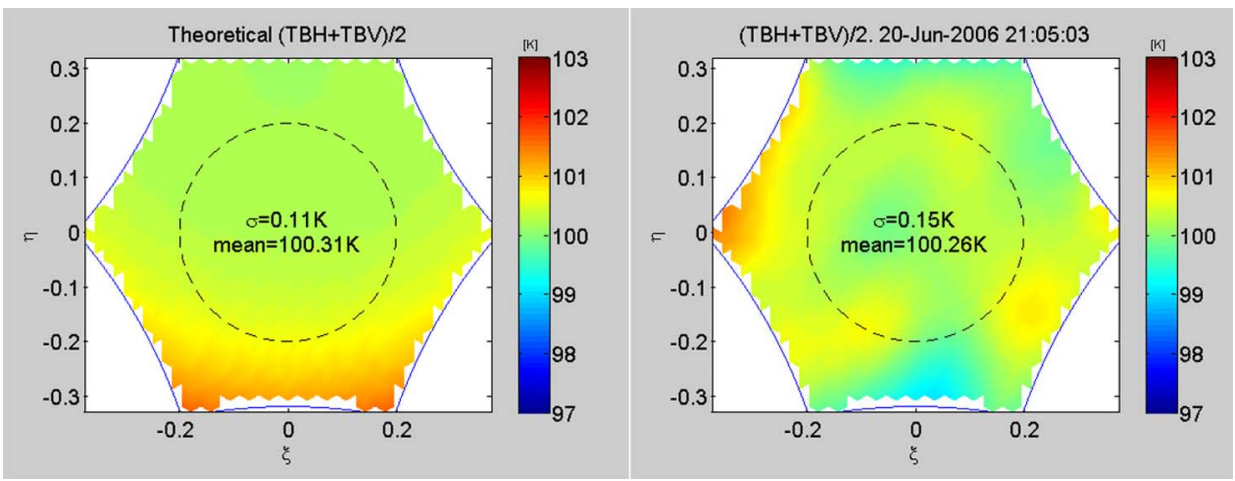

Fig. 7. Average of horizontal and vertical brightness temperature (half of first Stokes' parameter). (Left) Theoretical predictions using Fresnel reflection coefficients. (Right) Result of averaging 41 snapshots in the center of the lake.

option that produces consistent results is approach \#3, both with the FFT and the $G$ matrix. To understand the image, it should be noted that it is for vertical polarization ${ }^{2}$ and the bottom of the image corresponds to larger incidence angles. The increase in the brightness temperature is consistent with theoretical expectations. The artifacts seen are probably due to a bad compensation of the aircraft attitude.

Another interesting analysis is the choice of the flat-target response. Fig. 6 shows, for the water snapshot, the results of the $G$-matrix inversion using approach \#3 and selecting different flat-target responses. Based also on the visual results, the best choice, in this case, is the one retrieved from the cold-sky visibility measurement. It is interesting to point out that the option FTR $=0$ apparently produces, in this case, better results than the direct computation from antenna pattern measurements. This can be due to propagation of errors in the measurement of the antenna patterns and also to the fact that the FTR includes other effects, such as mismatch and fringe-washing function, that were not considered in the theoretical computation. The antenna patterns were measured in the facilities of the "Universitat Politècnica de Catalunya" (http://www.tsc.upc.edu/recerca/r_grups_amp.php?sel=recerca $\&$ sub $=$ grups\&idg=1\&amp=1) before the start of the flight campaign.

\footnotetext{
${ }^{2}$ This is defined in the instrument frame. No rotation has been implemented.
}

TABLE III

COMPARISON OF DIFFERENT APPROACHES USING FIRST STOKES' PARAMETER STATISTICS

\begin{tabular}{|c|c|c|c|}
\hline & \multicolumn{3}{|c|}{ Approach number } \\
\hline & 1 & 2 & 3 \\
\hline FFT & $\begin{array}{l}\text { mean }=116.9 K \\
\sigma=4.4 K\end{array}$ & $\begin{array}{l}\text { mean }=92.0 K \\
\sigma=2.35 K\end{array}$ & $\begin{array}{l}\text { mean }=100.2 K \\
\sigma=0.36 K\end{array}$ \\
\hline \multirow[t]{3}{*}{ G-matrix } & $\begin{array}{l}\text { mean }=126.1 K \\
\sigma=7.16 K\end{array}$ & $\begin{array}{l}\text { mean }=90.7 K \\
\sigma=3.83 K\end{array}$ & $\begin{array}{l}\text { mean }=100.3 K \\
\sigma=0.15 K\end{array}$ \\
\hline & \multicolumn{3}{|c|}{ FTR choice in approach number 3} \\
\hline & FTR $=0$ & Antenna meas & Cold Sky \\
\hline G-matrix & $\begin{array}{l}\text { mean }=97.7 K \\
\sigma=0.31 K\end{array}$ & $\begin{array}{l}\text { mean }=99.0 K \\
\sigma=0.93 K\end{array}$ & $\begin{array}{l}\text { mean }=100.3 \mathrm{~K} \\
\sigma=0.15 \mathrm{~K}\end{array}$ \\
\hline
\end{tabular}

\section{B. Quantitative Results}

The previous results are consolidated by using a parameter able to numerically compare the quality of the different approaches. The spatial variation of the first Stokes' parameter in a water retrieval has been used. This is a convenient choice because it is independent of field rotation and has a low dependence with respect to the incidence angle. Fig. 7 (left) shows the theoretical predictions using directly the Fresnel reflection coefficients. At right, the average of 41 snapshots, measured by the instrument and inverted using approach \#3 and the flat-target response from cold-sky measurement, is given. The spatial standard deviation is as low as $\sigma=0.15 \mathrm{~K}$, consistent with the theoretical value, although some artifacts are seen in the image. The spatial mean of the brightness temperature is the same in both. 
The spatial mean and standard deviation have been also computed for the same snapshots but using different approaches in inversion. The results are summarized in Table III, presented in the same order with that shown in Figs. 5 and 6. The qualitative results confirm the conclusions derived from just the visual inspection in the previous section. Note that the different approaches not only provide different values of standard deviation but that also the mean brightness temperature in the field of view is different. This confirms the predictions about the scene bias given in [9].

\section{CONCLUSION}

In synthetic aperture radiometers, there are several ways to combine visibilities, antenna temperatures, and instrument characterization data to get the final equation from which brightness-temperature maps are obtained. Three different approaches have been presented, and detailed equations for their implementation have been given. All of them are mathematically equivalent so that, in the absence of errors in instrument characterization data, they should all give the same results. However, antenna pattern uncertainties propagate differently in each of them, producing large differences in the final quality of the retrieved image. The use of incremental visibility reduces substantially the impact of these errors.

By analyzing real data provided by an airborne instrument, it has been shown that better images are obtained when the incremental visibility is computed by applying the "flat-target response" to the difference between the antenna temperatures and the receiver physical temperatures. This has been named as "approach \#3" and gives a visibility mathematically equal to zero at the origin. Its practical implementation depends on how the flat-target response is measured or estimated and what strategy was used to measure the different antenna temperatures. In the AMIRAS, best results were obtained when using a flattarget response measured by looking to the cold sky rather than theoretically computing from antenna pattern measurements, which performs even worse than just using a zero flat-target response. However, this conclusion highly depends on the quality of the antenna characterization data and may not be true for MIRAS in SMOS.

Two simple methods have been used to invert the equation, namely, an FTT using an average of all antenna patterns and a pseudoinverse of the $G$ matrix after averaging all elements of the matrix corresponding to redundant baselines. For the data analyzed, both methods perform similarly, although the $G$-matrix inversion is slightly better for approach \#3. It should be noted that the elements of the $G$ matrix are basically the antenna patterns; hence, the quality of this method is directly linked to the quality of the antenna characterization. Moreover, the fringe-washing function-which is included in the $G$-matrix elements-has a negligible effect in AMIRAS but may have an impact in larger instruments. In consequence, SMOS data will probably produce good images using FFT; however, the $G$-matrix inversion will be needed to comply with the quality requirements of level 1.

Finally, a proposal to compute an average pattern from the flat-target response is presented. In conjunction with the Fourier inversion, it allows one to obtain images without having to characterize individual antennas. It can be useful to get fast but good images in instruments not providing antenna patterns.

\section{REFERENCES}

[1] D. M. Le Vine and J. C. Good, Aperture Synthesis for Microwave Radiometers in Space. Greenbelt, MD: NASA Goddard Space Flight Center, Aug. 1983. Technical Memorandum TM-85033.

[2] D. M. Le Vine, C. T. Swift, and M. Haken, "Development of the synthetic aperture microwave radiometer, ESTAR," IEEE Trans. Geosci. Remote Sens., vol. 39, no. 1, pp. 199-202, Jan. 2001.

[3] P. Silvestrin, M. Berger, Y. Kerr, and J. Font, "ESA's second earth explorer opportunity mission: The soil moisture and ocean salinity missionSMOS," IEEE Geosci. Remote Sens. Newsl., no. 118, pp. 11-14, Mar. 2001.

[4] M. Martín-Neira and J. M. Goutoule, "MIRAS-A two-dimensional aperture-synthesis radiometer for soil moisture and ocean salinity observations," ESA Bull., vol. 92, pp. 95-104, Nov. 1997.

[5] K. Rautiainen, J. Kainulainen, T. Auer, S. Tauriainen, and M. Hallikainen, "Helsinki University of Technology synthetic aperture radiometerHUT-2D," in Proc. IGARSS, Barcelona, Spain, Jul. 23-27, 2007, pp. $3635-3638$.

[6] M. Martín-Neira, I. Cabeza, C. Pérez, M. A. Palacios, M. A. Guijarro, S. Ribó, I. Corbella, S. Blanch, F. Torres, N. Duffo, V. González, S. Beraza, A. Camps, M. Vall-llossera, S. Tauriainen, J. Pihlflyckt, J. P. González, and F. Martin-Porqueras, "AMIRAS - An airborne MIRAS demonstrator," IEEE Trans. Geosci. Remote Sens., vol. 46, no. 3, pp. 705716, Mar. 2008.

[7] B. Lambrigtsen, A. Tanner, T. Gaier, P. Kangaslahti, and S. Brown, "Developing a GeoSTAR science mission," in Proc. IGARSS, Barcelona, Spain, Jul. 23-27, 2007, pp. 5232-5236.

[8] J. Wu, C. Zhang, H. Liu, W. Sun, and J. Yan, "Clock scan of imaging interferometric radiometer and its applications," in Proc. IGARSS, Barcelona, Spain, Jul. 23-27, 2007, pp. 5244-5246.

[9] A. Camps, M. Vall-llossera, I. Corbella, N. Duffo, and F. Torres, "Improved image reconstruction algorithms for aperture synthesis radiometers," IEEE Trans. Geosci. Remote Sens., vol. 46, no. 1, pp. 146-158, Jan. 2008.

[10] E. Anterrieu, "On the reduction of the reconstruction bias in synthetic aperture imaging radiometry," IEEE Trans. Geosci. Remote Sens., vol. 45 , no. 3, pp. 592-601, Mar. 2007.

[11] I. Corbella, N. Duffo, M. Vall-llossera, A. Camps, and F. Torres, "The visibility function in interferometric aperture synthesis radiometry," IEEE Trans. Geosci. Remote Sens., vol. 42, no. 8, pp. 1677-1682, Aug. 2004.

[12] P. Moreno-Galbis, J. Kainulainen, and M. Martín-Neira, "Experimental demonstration of the Corbella Equation for aperture synthesis microwave radiometry," IEEE Trans. Geosci. Remote Sens., vol. 45, no. 4, pp. 945957, Apr. 2007.

[13] R. Butora, M. Martín-Neira, and A. L. Rivada-Antich, "Fringewashing function calibration in aperture synthesis microwave radiometry," Radio Sci., vol. 38, no. 2, pp. 15-1-15-15, Apr. 24, 2003. DOI: $10.1029 / 2002 R S 002695$.

[14] I. Corbella, F. Torres, A. Camps, A. Colliander, M. Martín-Neira, S. Ribó, K. Rautiainen, N. Duffo, and M. Vall-llossera, "MIRAS end-toend calibration. Application to SMOS L1 processor," IEEE Trans. Geosci. Remote Sens., vol. 43, no. 5, pp. 1126-1134, May 2005.

[15] I. Corbella, F. Torres, A. Camps, N. Duffo, and M. Vall-llossera, Polarimetric and Extended Formulation of the Visibility Function. Barcelona, Spain: Universitat Politècnica de Catalunya, Nov. 10, 2004. Technical note MP3-TN-UPC-3000-0002, rev.1.1.

[16] M. Martín-Neira, M. Suess, and J. Kainulainen, "The flat target transformation," IEEE Trans. Geosci. Remote Sens., vol. 46, no. 3, pp. 613-620, Mar. 2008.

[17] A. Camps, J. Bará, I. Corbella, and F. Torres, "The processing of hexagonally sampled signals with standard rectangular techniques: Application to 2D large aperture synthesis interferometric radiometers," IEEE Trans. Geosci. Remote Sens., vol. 35, no. 1, pp. 183-190, Jan. 1997.

[18] A. Camps, J. Bará, F. Torres, I. Corbella, and J. Romeu, "Impact of antenna errors on the radiometric accuracy of large aperture synthesis radiometers. Study applied to MIRAS," Radio Sci., vol. 32, no. 2, pp. 657668, Mar./Apr. 1997.

[19] A. Camps, I. Corbella, F. Torres, N. Duffo, M. Vall-llossera, and M. Martín-Neira, "The impact of antenna pattern dependence in aperture synthesis microwave radiometers," IEEE Trans. Geosci. Remote Sens., vol. 43 , no. 10 , pp. 2218-2224, Oct. 2005. 
[20] A. Tanner and C. T. Swift, "Calibration of a synthetic aperture radiometer," IEEE Trans. Geosci. Remote Sens., vol. 31, no. 1, pp. 257-267, Jan. 1993.

[21] E. Anterrieu and A. Khazaal, "Brightness temperature map reconstruction from dual-polarimetric visibilities in synthetic aperture imaging radiometry," IEEE Trans. Geosci. Remote Sens., vol. 46, no. 3, pp. 606-612, Mar. 2008

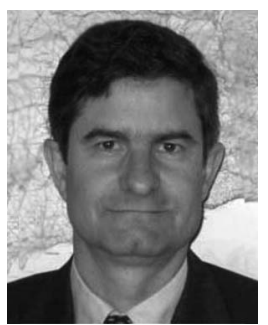

Ignasi Corbella (M'99-SM'08) received the Telecommunication Engineer and Dr.Eng. degrees from the Universitat Politècnica de Catalunya (UPC), Barcelona, Spain, in 1977 and 1983, respectively.

In 1976, he was with the School of Telecommunication Engineering, UPC as a Research Assistant with the Microwave Laboratory, where he worked on passive microwave integrated-circuit design and characterization. In 1979, he was with ThomsonCSF, Paris, France, where he worked on microwave oscillators' design. He has been with UPC since 1982, where he was first an Assistant Professor then as an Associate Professor in 1986 and, since 1993, as a Full Professor, where, from 1993 to 1997 he was an Academic Director with the School of Telecommunications Engineering, from 2001 to 2003 he was a Director with the Department of Signal Theory and Communications, and where he is currently teaching basic microwaves and antennas at the undergraduate level and graduate courses on nonlinear microwave circuits. Since 1993, he has been actively participating as a Researcher with the European Space Agency (ESA) Soil Moisture and Ocean Salinity (SMOS) mission in the frame of several contracts, directly with ESA or with the payload prime contractor EADS-Casa Espacio. His expertise includes, among others, fundamentals of interferometric aperture synthesis radiometry, image reconstruction algorithms, and onboard calibration, hardware specification, and payload characterization. From 1998 to 1999, he was with the NOAA/Environmental Technology Laboratory as a Guest Researcher developing methods for total-power radiometer calibration and data analysis. From 1999 to 2007, he was the Scientific Coordinator of a dictionary of telecommunication terms in the Catalan language, with more than 4000 entries, published in March 2007. Since 2004, he has been a Member of the SMOS Science Advisory Group and, since 2007, he has been a member of the SMOS Barcelona Expert Centre.

Dr. Corbella was the General Chairman of the 2007 International Geoscience and Remote Sensing Symposium in Barcelona.

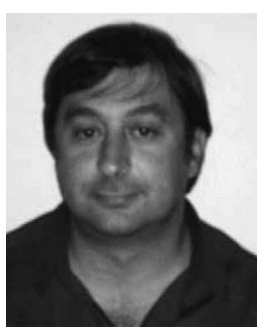

Francesc Torres (S'88-M'96-SM'06) was born in Ibiza, Spain, in 1962. He received the Ingeniero and Doctor Ingeniero degrees in telecommunication engineering from the Universitat Politècnica de Catalunya (UPC), Barcelona, Spain, in 1988 and 1992, respectively.

From 1988 to 1989 , he was a Research Assistant with the RF System Division, European Space Agency (ESA), Holland, The Netherlands, where he was devoted to microwave device testing and characterization. From 1989 to 1996, he was an Assistant Professor of microwave circuits and systems with the Signal Theory and Communications Department, Remote Sensing Laboratory, UPC, where he has been an Associate Professor since 1996. From 2005 to 2006, he held a sabbatical stage with the Microwave Systems Section, Jet Propulsion Laboratory, Pasadena, CA, where he was involved in the GeoSTAR pilot project, which is a passive microwave interferometric geosounder. Since 1995, he has been participating in a number of projects related to the Soil Moisture and Ocean Salinity (SMOS) mission by the ESA. He is currently coleading the Barcelona SMOS Expert Centre on Radiometric Calibration and Ocean Salinity.

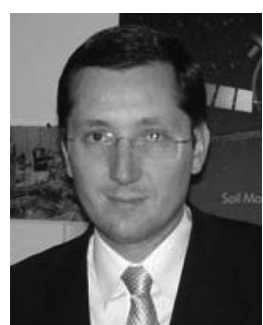

Adriano Camps (S'91-A'97-M'00-SM'03) was born in Barcelona, Spain, in 1969. He received the Telecommunication Engineer and the Dr.Eng. degrees from the Universitat Politècnica de Catalunya (UPC), Barcelona, Spain, in 1992 and 1996, respectively.

From 1991 to 1992, he was with the École Nationale Superieure (ENS) des Télécommunications de Bretagne, Brest, France, with an Erasmus Fellowship. Since 1993, he has been with the Electromagnetics and Photonics Engineering Group, Department of Signal Theory and Communications, UPC, where he was first an Assistant Professor, then, in 1997, was an Associate Professor, and, since 2007, has been a Full Professor. In 1999, he was on sabbatical leave at the Microwave Remote Sensing Laboratory, University of Massachusetts, Amherst. His research interests are focused on microwave remote sensing, with special emphasis on microwave radiometry by aperture synthesis techniques. He has performed numerous studies within the frame of European Space Agency Soil Moisture and Ocean Salinity Earth Explorer Mission, which have received several awards. He is the Associate Editor of Radio Science.

Dr. Camps was the recipient of the second national award of university studies in 1993, the INDRA award of the Spanish Association of Telecommunication Engineering to the best Ph.D. in Remote Sensing in 1997, the extraordinary Ph.D. award at UPC in 1999, the Research Distinction of the Generalitat de Catalunya for contributions to microwave passive remote sensing in 2002, and the EUropean Young Investigator Award in 2004. In addition, as a member of the Microwave Radiometry Group at UPC, he was the recipient, in 2000, 2001, and 2004, of the First Duran Farell and the Ciudad de Barcelona awards for Technology Transfer and of the "Salvà I Campillo" Award of the Professional Association of Telecommunication Engineers of Catalonia for the most innovative research project, respectively. He was the Chair of $\mu \mathrm{Cal}$ ' 01 . From 2003 to 2006, he was an Editor of the IEEE GEOSCIENCE AND REMOTE SEnSing Newsletter and, currently, is the Associate Editor of the IEEE Geoscience and Remote Sensing Letters. He is the President-Founder of the IEEE Geoscience and Remote Sensing Society Chapter, Spain.

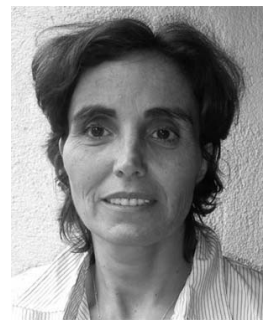

Nuria Duffo (S'91-M'95) received the Telecommunication Engineering and Ph.D. degrees in telecommunication engineering from the School of Telecommunications Engineering, Universitat Politècnica de Catalunya (UPC), Barcelona, Spain, in 1990 and 1996, respectively.

Since 1997, she has been an Associate Professor with the Department of Signal Theory and Communications, UPC. Her current research activities include numerical methods in electromagnetics, microwave radiometry, antenna analysis, and design.

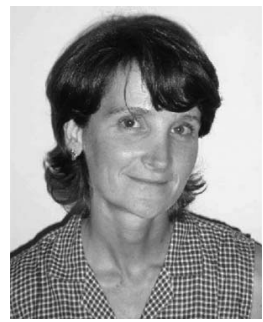

Mercè Vall-llossera (M'99) received the Senior Telecommunication Engineering and the Ph.D. degrees in telecommunication engineering from the Universitat Politècnica de Catalunya (UPC), Barcelona, Spain, in 1990 and 1994, respectively.

She has been lecturing and doing research with the Department of Signal Theory and Communications, UPC, as an Assistant Professor from 1990 to 1997 and as an Associate Professor, since 1997. She spent a sabbatical year in Montreal with the scholarship of the "Programme Quebecois de Bourses d'excellence (1996.1997): Stages de Formation postdoctorale au Quebec pour jeunes diplomes etrangers." Her research activities are numerical methods in electromagnetics, microwave radiometry, antenna analysis, and design. Currently, her research is mainly related to the study of numerical methods applied to sea surface emissivity and their characterization at L-band and the Microwave Imaging Radiometer with Aperture Synthesis/Soil Moisture and Ocean Salinity project.

Dr. Vall-llossera was the recipient of the "Primer Premio Duran Farell de Investigación Tecnológica," with the other member of the Radiometry Group at UPC, in 2000, and the "Primer Premio Ciutat de Barcelona d'Investigació Tecnòlogica" in 2001. 